\title{
Renormalization of Polyakov loops in fundamental and higher representations
}

\author{
Olaf Kaczmarek* \\ Fakultät für Physik, Universität Bielefeld, D-33615 Bielefeld, Germany \\ E-mail: okaczephysik.uni-bielefeld.de
}

\section{Sourendu Gupta}

Department of Theoretical Physics, Tata Institute for Fundamental Research, Homi Bhabha Road, Mumbai 400 005, India

\section{Kay Hübner}

Physics Department, Brookhaven Natl. Laboratory, Upton, New York 11973, USA

\begin{abstract}
We compare two renormalization procedures, one based on the short distance behavior of heavy quark-antiquark free energies and the other by using bare Polyakov loops at different temporal extent of the lattice and find that both prescriptions are equivalent, resulting in renormalization constants that depend on the bare coupling. Furthermore these renormalization constants show Casimir scaling for higher representations of the Polyakov loops.

The analysis of Polyakov loops in different representations of the color SU(3) group indicates that a simple perturbative inspired relation in terms of the quadratic Casimir operator is realized to a good approximation at temperatures $T \gtrsim T_{c}$ for renormalized as well as bare loops.

In contrast to a vanishing Polyakov loop in representations with non-zero triality in the confined phase, the adjoint loops are small but non-zero even for temperatures below the critical one. The adjoint quark-antiquark pairs exhibit screening. This behavior can be related to the binding energy of gluelump states.
\end{abstract}

The XXV International Symposium on Lattice Field Theory

July 30-4 August 2007

Regensburg, Germany

\footnotetext{
* Speaker.
} 

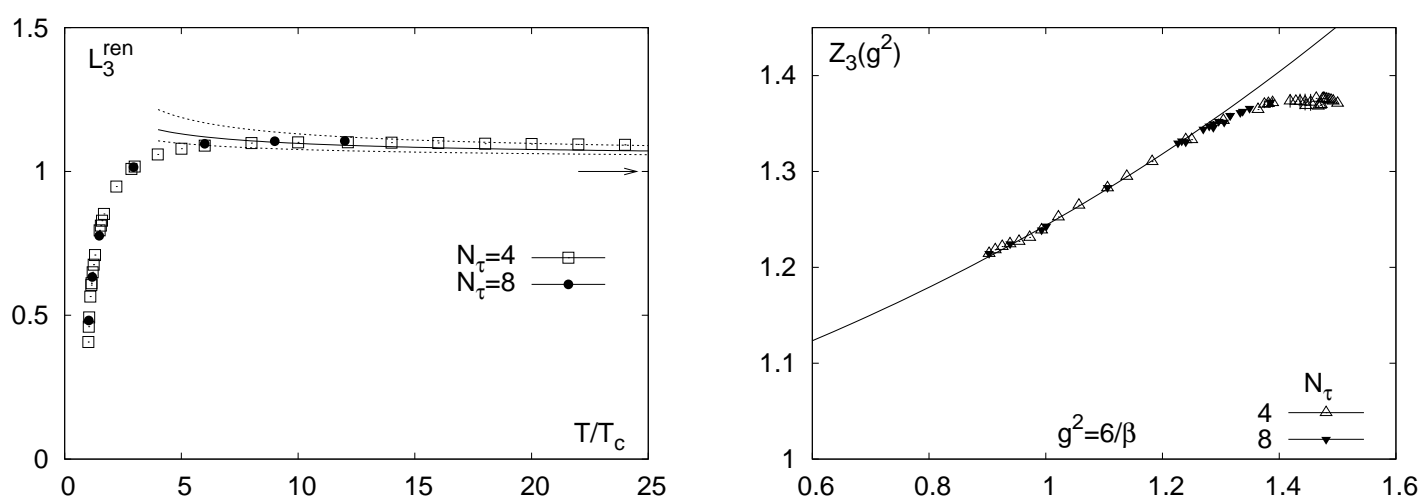

Figure 1: Renormalized fundamental Polyakov loop (left) and renormalization constants (right) in SU(3) pure gauge theory for two values of the temporal lattice extent $N_{\tau}$. The lines in the left figure show the perturbative result [1, 2] .The arrow represents the asymptotic high temperature limit, $L^{\text {ren }}=1$. The line in the right figure shows a perturbative inspired fit.

\section{Introduction}

Studies of the transition from a confined to a deconfined medium as well as the fundamental question for a proof of confinement are strongly related to the Polyakov loop. Models based on the Polyakov loop are proposed to describe the transition to a quark gluon plasma phase and its properties at zero as well as non-zero baryon density in a phenomenological manner [3, 4, 5, 6, 7, 8, 9, 10, 11, 12, 13,. Furthermore the connection of SU(3) theory to the large $N_{c}$-limit (in a mean-field approximation) is widely discussed [ [ 4 , 5.

For a test of the reliability and comparison of these models to pure gauge theory and QCD with dynamical quarks, a detailed knowledge of the behavior of the renormalized Polyakov loop in the fundamental and higher representations in those theories is of fundamental importance.

We will present two different renormalization procedures for the Polyakov loop for different representations, show their equivalence and discuss our main results of this study in pure SU(3) gauge theory.

\section{Fundamental and adjoint Polyakov loops}

The renormalization of Polyakov loops (in the fundamental representation) using the short distance behavior of static quark-antiquark free energies was outlined in [14]. For arbitrary representations of the static sources this can be written as,

$$
e^{-F_{D}^{1}(r, T) / T}=\left(Z_{D}\left(g^{2}\right)\right)^{2 d_{D} N_{\tau}}\left\langle\operatorname{Tr}\left(L_{D}(\vec{x}) L_{D}^{\dagger}(\vec{y})\right),\right.
$$

which is equivalent to the renormalization of the Polyakov loop itself,

$$
L_{D}^{r e n}=\left(Z_{D}\left(g^{2}\right)\right)^{N_{\tau} d_{D}}\left\langle L_{D}^{\text {bare }}\right\rangle .
$$

The renormalization constants are obtained by matching the free energies to the zero temperature potential at short distances. In fig. 1 1 we show the results for the renormalized Polyakov loop (left) 

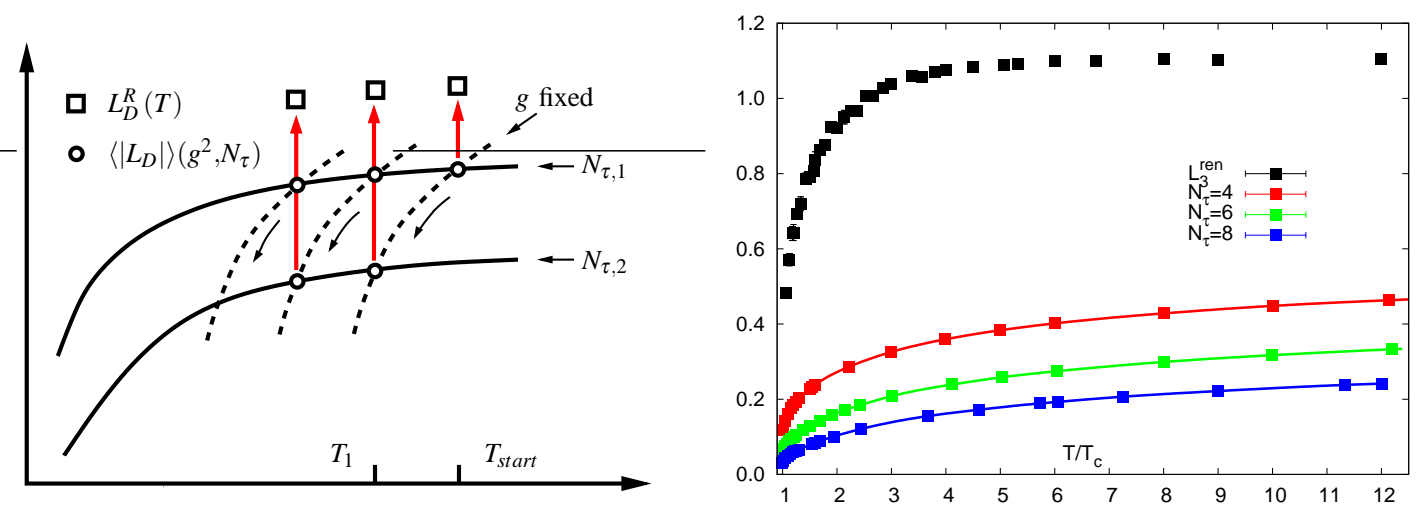

Figure 2: Renormalization procedure using different $N_{\tau}$ (left). Bare Polyakov loops from $32^{3} \times N_{\tau}$ lattices and the resulting $L_{3}^{r e n}$. The lines are spline interpolations (right).
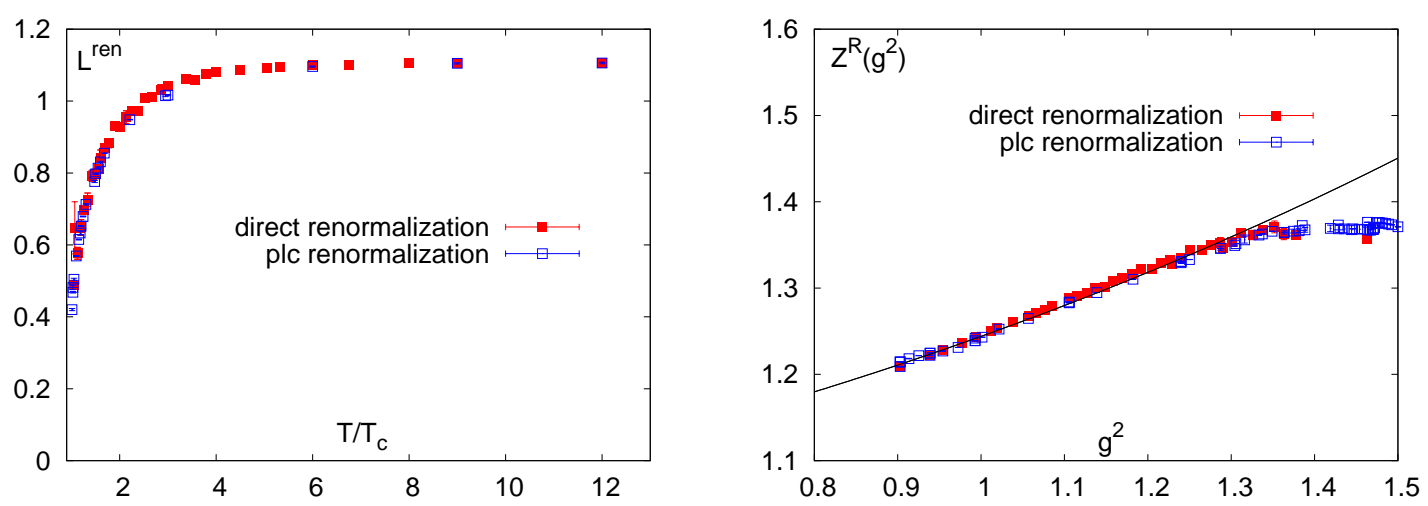

Figure 3: Comparison of the renormalized Polyakov loop (left) and the renormalization constants (right) obtained with the two different renormalization procedures.

and the renormalization constants (right) for two different $N_{\tau}$ obtained in quenched QCD. The good agreement of $Z_{3}\left(g^{2}\right)$ and $L_{3}(T)$ for the $N_{\tau}=4$ and 8 indeed shows that the renormalization constants depend only on the bare coupling constants. In perturbation theory Casimir scaling for heavy quark potentials is realized (at least) up to two-loop order [15, 16].

\section{Direct renormalization in higher representations}

Using the observation that the renormalization constants depend only on the bare couplings opens the possibility for a direct renormalization procedure based on single bare Polyakov loops at different $N_{\tau}$ rather than using Polyakov loop correlation functions (a similar method was proposed in [17]).

The fist step in this procedure is to fix the arbitrary overall scale factor by fixing the value of the renormalized Polyakov loop at the highest temperature in our analysis, $T_{i}=T_{\max }=12 T_{c}$, where we use the same scheme as in the previous method. From this we obtain the renormalization constants 

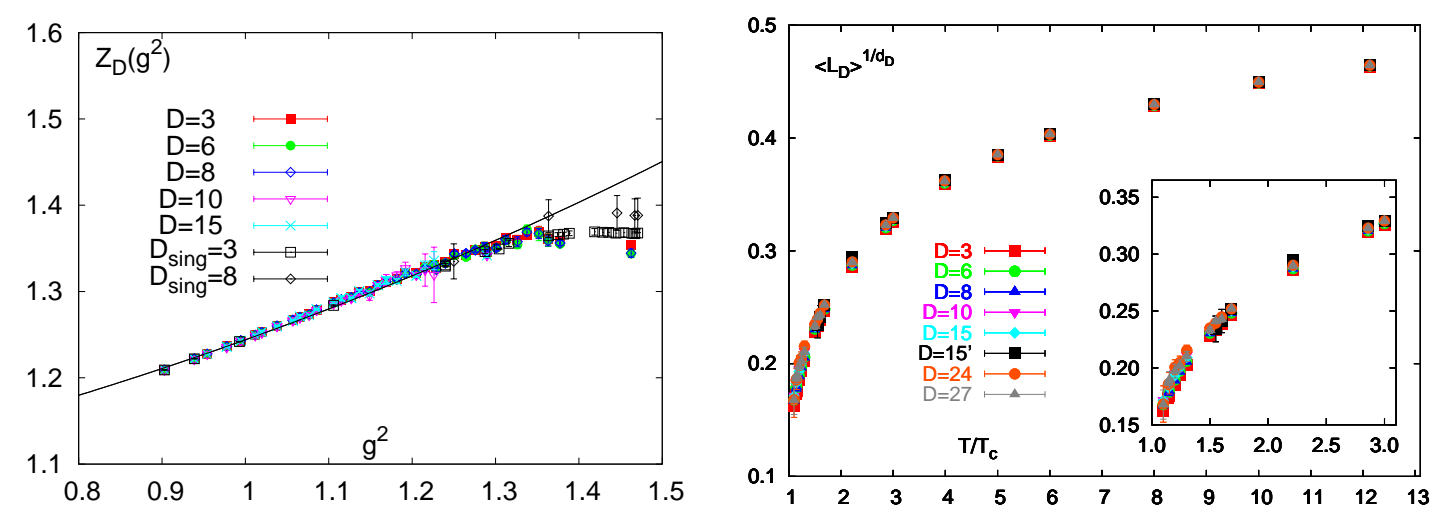

Figure 4: Renormalization constants obtained with the direct renormalization procedure (left). Also shown are the results obtained from the previous method for fundamental and adjoint loops, labeled $D_{\text {sing. }}$. Casimir scaled bare Polyakov loops (right) for different representations $D$.

at the corresponding coupling (at two different $N_{\tau}$ ) by assuming

$$
\begin{aligned}
& \left.Z_{D}^{d_{D} N_{\tau, i}}\left(g_{i}^{2}\right) L_{D}^{\text {bare }}\left(g_{i}^{2}, N_{\tau, i}\right)\right|_{\frac{1}{a\left(g_{i}^{2}\right) N_{\tau, i}}}=T_{i} \equiv L_{D}^{r e n}\left(T_{i}\right) \quad \text { and } \\
& \left.Z_{D}^{d_{D} N_{\tau, j}}\left(g_{j}^{2}\right) L_{D}^{\text {bare }}\left(g_{j}^{2}, N_{\tau, j}\right)\right|_{\frac{1}{a\left(g_{j}^{2}\right) N_{\tau, j}}}=T_{i} \equiv L_{D}^{r e n}\left(T_{i}\right) .
\end{aligned}
$$

This procedure can now be iterated (see fig. 2(left)) to obtain the renormalization constants and the renormalized Polyakov loop down to $T_{c}$. In fig. (2)(right) we show the result of this procedure for the fundamental loop in $\mathrm{SU}(3)$ pure gauge theory obtained by applying this procedure for three values of $N_{\tau}$.

The comparison of the two renormalization procedures (fig. 3) indeed shows that the renormalized Polyakov loops (left) and the renormalization constants (right) are in good agreement and both procedures are equivalent.

The prescription can easily be extended to Polyakov loops in any representation $D$ [18, 19], thus giving the renormalized Polyakov loops $L_{D}^{R}$ and the renormalization constants $Z_{D}\left(g^{2}\right)$. Using these, one can then check Casimir scaling in the form

$$
Z_{D}\left(g^{2}\right)=Z_{3}\left(g^{2}\right)
$$

for the renormalization constants and

$$
L_{D}^{r e n}(T)=\left(L_{3}^{r e n}(T)\right)^{d_{D}}
$$

for the Polyakov loops, where $d_{D}=C_{2}(D) / C_{2}(3)$ is the ratio of quadratic Casimirs. The test of Casimir scaling is then the independence of $Z$ from $D$. Note that (3.3) together with (2.2) implies that, if Casimir scaling is realized for the renormalized Polyakov loop, it holds for the bare loops as well.

In fig. $甘$ we show the results for the renormalization constants (left) and the Casimir scaled bare Polyakov loops (right) for representations up to $D=15$. For comparison we also include the results obtained from the previous method. We observe a good agreement of $Z_{D}$ for all representations in the whole coupling range and for the scaled Polyakov loops for temperatures down to the critical one. 

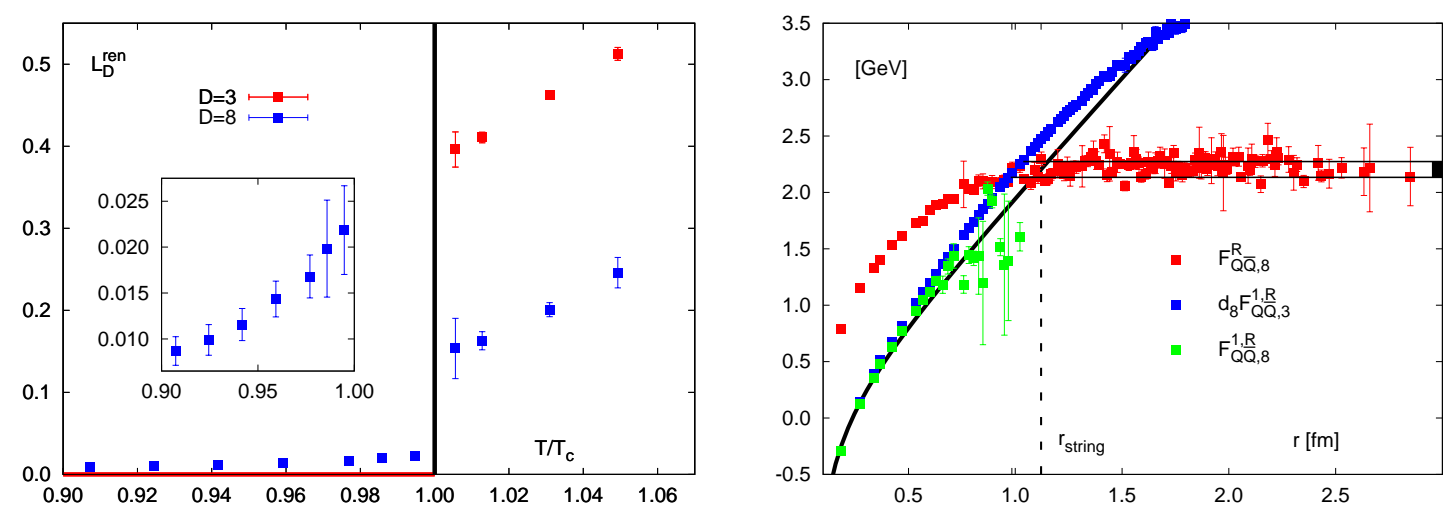

Figure 5: Renormalized adjoint Polyakov loop compared to the fundamental loops (left). Heavy quarkantiquark free energies for adjoint sources in the color singlet and color averaged channel compared to Casimir scaled color singlet free energy of fundamental sources at $T=0.959 T_{c}$. The lines show the asymptotic value and estimates for the string breaking distance.

\section{Adjoint Polyakov loops and string breaking}

In contrast to Polyakov loops with non-zero triality, which have vanishing expectation values in the confined phase (in the infinite volume limit), for all triality-zero representations $(\mathrm{r}=8,10,27, \ldots)$ one expects to see string breaking below $T_{c}$ also in pure gauge theory, and hence a non-vanishing Polyakov loop in the infinite volume limit (see also discussions in [20, 21, 22]).

We have computed the infinite volume, renormalized adjoint Polyakov loop below $T_{c}$. Fig. (1) (left) shows the results compared to the fundamental loop around $T_{c}$. While the fundamental renormalized Polyakov loop is zero below $T_{c}$, the adjoint loop is small but clearly non-vanishing.

For the other triality-zero representations $(r=10,27)$ we expect the same behavior, but we cannot give the infinite volume limit below $T_{c}$, since the corresponding data is still too noisy for the statistics achieved in this work.

For the heavy quark-antiquark free energies of adjoint sources we observe string breaking below $T_{c}$ (fig. 5 (right)). The asymptotic value of the static quark-antiquark free energy of adjoint sources (fig. 6) can be related to the binding energy of gluelump states [23], i.e. bound states of a dynamical gluon with a static adjoint source. In the upper part of fig. 6 we show the results for the asymptotic values of the adjoint heavy quark free energies and in the lower part an estimate for the string-breaking radius defined through

$$
V_{8}\left(r_{\text {string }}\right)=F_{8}(r=\infty, T)
$$

where $V_{8}$ is the zero temperature potential and $F_{\infty}$ is the asymptotic value of the quark-antiquark free energy, both for adjoint sources.

An extension of this study will be the analysis of color octet states of heavy quark-antiquark free energies with fundamental sources combined with a static adjoint source, forming a color singlet state in total. 


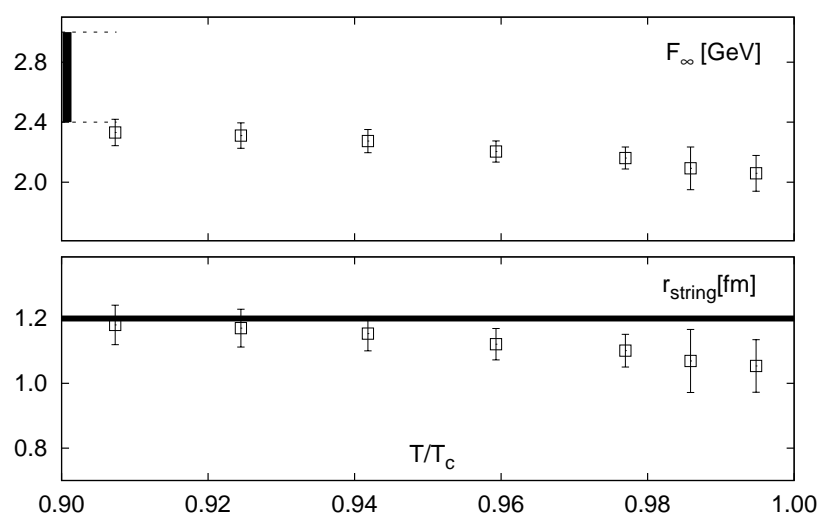

Figure 6: Asymptotic value of the adjoint heavy quark free energies (upper panel). Estimate of the string breaking radius (lower panel).

\section{Conclusions and Outlook}

We have extended the renormalization procedure outlined in [14] to quark sources in the adjoint representation. We observed that the resulting renormalization constants only depend on the bare coupling $g^{2}$. This led to the proposal of a new (direct) renormalization procedure for the Polyakov loop itself measured at different temporal lattice extent in contrast to the (indirect) renormalization using two-point correlation functions of Wilson lines or Polyakov loops.

We have shown that both procedures are indeed equivalent leading to a solid description of the renormalized Polyakov loop. Furthermore we applied the new prescription to Polyakov loops in the fundamental and higher representations up to $D=27$.

The direct renormalization procedure is solely based on gauge invariant quantities, while the $q \bar{q}-$ renormalization is based on color singlet correlation functions of Wilson lines which are (in principle) gauge dependent quantities. The equivalence of both procedures, i.e. the agreement of the renormalization constants and the renormalized Polyakov loops, shows that (at least) the short (temperature independent) as well as the (asymptotic) large distance part of the heavy quark free energies obtained in Coulomb gauge become gauge independent as proposed in [24, 25].

The analysis of Polyakov loops in higher representations up to $D=27$ led to the the observation that Casimir scaling for the Polyakov loops and the corresponding renormalization constants in different representations is a surprisingly good approximation even down close to $T_{c}$. This may indicate that non-Casimir scaling terms in a perturbative series may only play a sub-dominant role. Due to the Z(3)-symmetry of the pure gauge theory, all Polyakov loops with non-zero triality vanish in the confined phase even in the absence of dynamical quarks, i.e. pure gauge theory. For the adjoint representation we have observed small, but non-zero values below $T_{c}$. The static adjoint sources can couple to the dynamical adjoint constituents (gluons) of the theory and the quarkantiquark pair gets screened even in the confined phase. This screening phenomenon (string breaking) is visible in the heavy quark free energies which have a finite asymptotic value while for zero-zero triality they rise linearly with distance. The finite asymptotic value for adjoint sources may be related to the binding energy of gluelump states. 
A more detailed study and discussion of the renormalization of Polyakov loops in higher representations as well as the application to QCD with dynamical quarks is in preparation [19]. A future extension of this study will be the analysis of correlation functions of different representations, e.g. a baryonic system made up of a color octet state of a quark-antiquark pair in the fundamental representation combined with a static adjoint source forming a color singlet state in total.

\section{Acknowledgment}

This work has been supported by Contract No. DE-AC02-98CH10886 with the U. S. Department of Energy. At an early stage of this work K. H. has been supported by the DFG under grant GRK 881/1. S. G. would like to acknowledge the hospitality of the University of Bielefeld.

\section{References}

[1] E. Gava and R. Jengo, Phys. Lett. B105 (1981) 285.

[2] A. M. Polyakov, Nucl. Phys. B164 (1980) 171.

[3] R. D. Pisarski, Phys. Rev. D74 (2006) 121703.

[4] A. Dumitru, Y. Hatta, J. Lenaghan, K. Orginos, and R. D. Pisarski, Phys. Rev. D70 (2004) 034511.

[5] A. Dumitru, J. Lenaghan, and R. D. Pisarski, Phys. Rev. D71 (2005) 074004.

[6] A. Dumitru, R. D. Pisarski, and D. Zschiesche, Phys. Rev. D72 (2005) 065008.

[7] A. Dumitru, D. Roder, and J. Ruppert, Phys. Rev. D70 (2004) 074001.

[8] E. Megias, E. Ruiz Arriola, and L. L. Salcedo, JHEP 01 (2006) 073.

[9] E. Megias, E. Ruiz Arriola, and L. L. Salcedo, Phys. Rev. D74 (2006) 065005.

[10] C. Ratti, M. A. Thaler, and W. Weise, Phys. Rev. D73 (2006) 014019.

[11] P. N. Meisinger, M. C. Ogilvie, and T. R. Miller, Phys. Lett. $B 585$ (2004) 149.

[12] K. Fukushima, Phys. Rev. D68 (2003) 045004.

[13] D. Diakonov and M. Oswald, Phys. Rev. D70 (2004) 105016.

[14] O. Kaczmarek, F. Karsch, P. Petreczky, and F. Zantow, Phys. Lett. B543 (2002) 41.

[15] Y. Schröder, Phys. Lett. B447 (1999) 321.

[16] G. S. Bali and P. Boyle, hep-lat/0210033.

[17] M. Creutz, Phys. Rev. D 23 (1981) 1815.

[18] S. Gupta, K. Hübner and O. Kaczmarek, Nucl. Phys. A 785 (2007) 278.

[19] S. Gupta, K. Hübner and O. Kaczmarek, in preparation.

[20] P. H. Damgaard, Phys. Lett. $\mathbf{B} 194$ (1987) 107.

[21] K. Redlich, H. Satz and J. Seixas, Phys. Lett. B208 (1988) 291.

[22] J. Fingberg, D. E. Miller, K. Redlich, J. Seixas and M. Weber, Phys. Lett. B248 (1990) 347.

[23] G. S. Bali and A. Pineda, Phys. Rev. D69 (2004) 094001.

[24] O. Philipsen, Phys. Lett. $\mathbf{B 5 3 5}$ (2002) 138.

[25] O. Jahn and O. Philipsen, Phys. Rev. D70 (2004) 074504. 